\title{
Observations on the design and specification of a wrist-worn human activity monitoring system
}

\author{
DANIEL P. REDMOND and FREDERICK W. HEGGE \\ Walter Reed Army Institute of Research, Washington, DC
}

\begin{abstract}
Monitoring motor activity provides an important index of sleep, rest, and activity in field studies of sustained operations, shift-work schedules, and sleep deprivation. Poor results with previous methods led to development of a program to design a technologically improved monitoring system. In this 3-year program, specific issues were examined, ranging from the empirical characteristics of the wrist-movement signal and transduction methods to conversion of that signal to a useful index of motility. In this report, we discuss the several design issues encountered as well as observations, conclusions, and resulting specifications. The product of this program is a microprocessor-controlled, self-contained activity recording system, with $16 \mathrm{~K}$ of digital storage and an operating life of over 30 days. The Walter Reed Activity Monitoring System is designed to examine further the behavioral and physiological correlates of activity.
\end{abstract}

There are various useful applications for the monitoring of general activity of unconfined human subjects. Devices that record motility are used to produce data series that index the daily cycle of rest and activity in individuals, variations in circadian rhythm, relative activity within days (ultradian effects) and across days (infradian effects), and the timing, duration, and disruption of sleep. Such data series have proved important in assessment of shift-work schedules; work in continuous operations scenarios; time-zone changes; hyperactivity and motor disorders in children; unipolar, bipolar, and seasonal affective disorders; and hypnotic, anxiolytic, and antidepressive drug effects (e.g., Borbeley, Loepfe, Mattman, \& Tobler, 1983; Kupfer et al.,1974; Porrino et al., 1983; Wehr, Sack, Rosenthal, Duncan, \& Gillin, 1983; Wever, 1981).

Over the last 20 years, the design technology of selfcontained, nonrestrictive activity monitors has improved considerably. Initial designs were modified wristwatches that showed an accumulated count of movements (Bell, 1968). In 1972, Foster et al. introduced an electronic telemetric device that permitted continuous recording; however, recording was restricted by the location of the receiver/recording apparatus. By the mid-1970s, McPartland, Kupfer, and Foster (1976) refined the monitors into

Acknowledgement is hereby made to David R. Thorne, Helen C. Sing, and Joseph Fritz. We also wish to acknowledge the valuable consultation of M. H. Loew and M. F. Eisenberg, of George Washington University, and the tireless efforts of Robert Conlan and Robert Williams of Precision Control Design, Inc. Their work was achieved through a contract awarded by the U.S. Army Medical Research and Development Command (Contract No. DAMD-17-83-Q-0027.

The opinions or assertions contained herein are the private views of the authors and are not to be construed as official or as reflecting the views of the Department of the Army or the Department of Defense (para 4-3, AR 360-5).

The authors' mailing address is: Department of Behavioral Biology, Walter Reed Army Institute of Research, Washington, DC 20307-5100. truly self-contained activity counters, with integrated circuitry and memory to provide internal control and timing as well as off-line data retrieval. Concurrently, Colburn, Smith, Guarini, and Simmons (1976) produced a similar system with different transducer and timing characteristics and 256 bytes of memory. Other activity monitors have consisted of transducers (wrist-worn or otherwise) connected by cable to light-weight tape recorders (Kripke, Mullaney, Messin, \& Wyborney, 1978 ) or to solid-state memory packages (Taylor et al., 1982). Wong, Webster, Montoye, and Washburn (1981) clarified the design issues surrounding accelerometric transducers used in this application. And most recently, Guening and Eugene (1985) described a design which incorporates microprocessor, read-only memory (ROM), and random-access memory (RAM) chips for programmable timing and storage functions, and a novel transducer design.

A main goal of our laboratory is to study rest/activity cycles and other behavioral variables of soldiers involved in sustained operations and other conditions that adversely affect sleep and performance. Large numbers of individuals are studied simultaneously, and operations occur in the field rather than in the laboratory environment. For several years we have monitored behaviors using activity monitors, similar to those designed by Colburn et al. (1976), along with commercial monitors (Vitalog Model PMS-8). Success was limited by the inconsistency and the narrow dynamic range of data, by artifact sensitivity, and by device maintenance problems. Examination of existing devices and their functions led us to conclude that serious flaws in design concept and technological limitations restricted the usefulness of the devices in our projects.

Three years ago, we began a program to optimize the design and utility of an activity monitoring system which we could then contract out for final development and manufacture. First, we studied the fundamental characteristics of the wrist-movement signal, from which a mo- 
tor activity index was derived. In an extension of work previously done under contract by Kripke and his colleagues (Webster, Messin, Mullaney, \& Kripke, 1982), we examined transducers, their responses to movements, analog signal processing, and signal-to-count transforms. Finally we outlined and specified a design.

\section{METHOD}

\section{Analog Recordings of Wrist Activity}

To acquire raw, undigitized movement signals for examination of signal properties, we mounted commercially acquired miniature accelerometers within wristwatch cases, which the subject wore on the wrist. The 36-in. transducer leads were threaded up the arm and down to a belt-worn miniature tape recorder (Oxford Medilog Model 4-24). A time base was provided by a $60-\mathrm{Hz}$ signal recorded on Channel 4 (Medilog ATE-1 module).

Preliminary studies were performed using transducers (Wilcoxon Research Model 137) and recording modules (Medilog AD-2). The linear frequency response, in the range of $0.5-40 \mathrm{~Hz}$, provided initial definitions of the movement signal. Thirty $24-\mathrm{h}$ tape recordings were obtained from soldiers on a training exercise. Repeated power spectral density (PSD) curves were computed, and 5,000 spectra were examined and averaged. Over $99 \%$ of signal power was produced in the frequency band below $5 \mathrm{~Hz}$. Individual instances of spectral peaks above $5 \mathrm{~Hz}$ occurred, but as isolated occurrences when the raw signal and logged activity suggested proximity to sources of external vibration, such as an operating automobile or lawn mower. Most of the signal power indicated that a system with lower frequency responses was both adequate and necessary for further studies of the signal.

For most observations reported in this paper, wrist activity was sensed by transducers [Entran Models EGA1F5D (single axis) and EGA3F5D (triple axis)] with a linear response from dc to above $100 \mathrm{~Hz}$ with a nominal sensitivity of $5.4 \mathrm{mV} / \mathrm{g}$. Input impedance was $1,000 \Omega$, requiring $5.4 \mathrm{~mA}$ of supply current, and output impedance was $500 \Omega$, obviating the need for special shielding or preamplification. Power was supplied by Medilog 4-24 recorder batteries, using a jumper on the ATE-1 time base module to provide a negative supply at Pin $\mathbf{H}$ of the input connector. Output was coupled to the inputs of a Medilog AM4 Carrier Modulation Recording Amplifier, which has a frequency response of dc to $10 \mathrm{~Hz}$. Calibration was achieved by rotating the transducer $90^{\circ}$, from horizontal to vertical, for a 1 -g output change. Gain of the AM4 was adjusted to produce an output of $1 \mathrm{~g}=1 / 2$ full scale, using a Medilog XM-2 Monitor Unit. Playback of data required a Medilog PM-3 Replay Demodulator Amplifier with a $500-\mathrm{Hz}$ low-pass filter, to provide a final passband of dc to $8.3 \mathrm{~Hz}$ at $60 \times$ playback speed. Gain was set to maximum, to yield an output of $0.25 \mathrm{~V} / \mathrm{g}$ for the calibrated recordings, and offset controls were adjusted to center the signals at $0 \mathrm{~V}$.
Three axis recordings utilized three identical recording channels in the same tape recorder. Orthogonal $x, y$, and $\mathrm{z}$ axes of the transducer were aligned on the wrist such that the $x$-axis was transverse (medial-lateral), the $y$-axis transecting (anterior-posterior), and the z-axis longitudinal with respect to the wrist.

\section{Signal Data Acquisition and Computations}

The activity signals retrieved on playback were filtered through adjustable bandpass filters (Coulbourn Model S75-36), set appropriately for each study. These outputs were then coupled to the inputs of a 4-channel, programmable digital oscilloscope, with $128 \mathrm{~K}$ wordbuffer memory for each channel (Norland Model 3001A). Analog-to-digital (A/D) conversion, with 10-bit resolution, occurred at a sampling rate derived by dividing the $60-\mathrm{Hz}$ recorded time base (e.g., by 4 for a $15-\mathrm{Hz}$ sampling rate). Programmability of the oscilloscope includes a variety of mathematical and statistical functions that can be performed on prescribed sets of the stored data. Using standard formulas, the root mean square (RMS), power spectral density (PSD) functions, mean, standard deviation, and linear correlations were performed where indicated. In a typical usage, a $15-\mathrm{Hz}$ sampling rate produced $128 \mathrm{~K}$ samples (or $145.64 \mathrm{~min}$ of activity recording), and 24-h tapes were studied in 10 successive sections. In blocks of 1,024 (or 1K) samples, RMS was computed for each $68.3 \mathrm{sec}$ of data; as an estimate of total power in the voltage waveform, these RMS values were used as activity scores for each block or epoch, and served as a standard against which other activity indices were compared. PSD functions were likewise computed from $1 \mathrm{~K}$ sized blocks of data and were averaged by summing for successive blocks.

\section{Tape-Recorded Studies of Wrist Activity}

The analog tape-recording system has been used in a variety of laboratory and field studies, including studies of soldiers in training exercises, temporal isolation (" free running"') experiments, and work-motion studies. Over 200 of these tapes have been examined for signal characteristics, and selected portions of these tapes are used in this report for illustrative purposes.

To summarize the variety of surveys performed on these data over the last 3 years, a special 2-h study was performed, using 1 subject engaged in a set schedule of activities, ranging from bed rest to reading, typing, walking, jogging, and vigorous calisthenics. The purpose of the study was to encompass a range of typical activity within a period convenient to the analytical and graphic methods applied in this report. Figure 1 depicts that 2-h session and the activities involved.

\section{Sources of the Wrist-Activity Signal}

An accelerometric signal derived from the wrist may be considered to emanate from several sources: (1) rotation of the transducer in relation to the vertical gravita- 


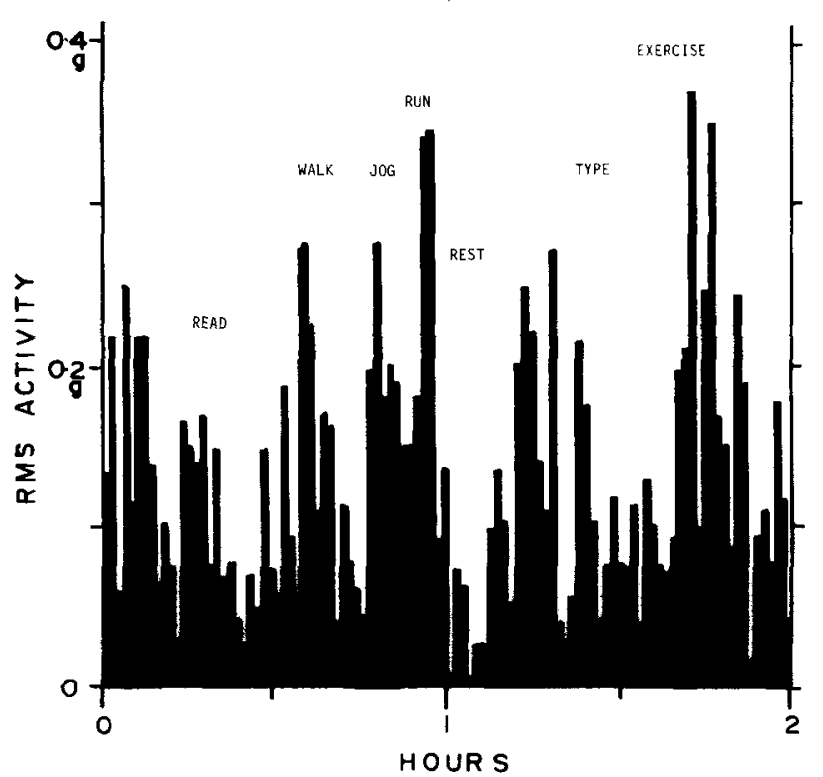

Figure 1. Two-hour activity session, tape recorded. Activity values represent root mean square (RMS) for each 68-sec epoch of the calibrated signal. Passband $=0.25-2.0 \mathrm{~Hz}$, sampling rate $=15 \mathrm{~Hz}$.

tional force; (2) changing velocity of the transducer in space due to displacement of the arm; (3) abrupt accelerations due to bounding or bumping of the transducer against other objects; (4) excitation of an undamped transducer at its frequency of mechanical resonance, also associated with bumping or jolting; (5) external vibrations and sounds coupled to the transducer; (6) electromagnetic noise interference; and (7) nonlinearities introduced by a viscous or freely moving object within the transducer (e.g., balls of mercury or steel). Of these, only the first two sources seem directly related to movement of the wrist (except insofar as proximity to or generation of noise might be considered an index of normal human activity). In bench testing of several candidate transducers, sources 3-7 were observed to dominate the transducers' responses, even among devices in standard usage. The analog recording system used for studies described here effectively rejected or attenuated such sources and, thus, was useful for examining characteristics of the signal associated with movement per se.

Figure 2 illustrates the complexity of the activity signal taken from the wrist, even with a frequency passband restricted to below $8.3 \mathrm{~Hz}$. This 68 -sec block of data was recorded in three axes while the subject was walking briskly (about 2 paces/sec); in addition to swinging his arms in the cadence of his gait, the subject was observed to move his arm about, check the wristwatch, and wipe his brow. In the last $30 \mathrm{sec}$ of the tracings, the walking cadence is most discernible (Component A) in the $\mathrm{Y}$-axis, fluctuating about a baseline of about $-1 \mathrm{~g}$, with a peakto-peak amplitude of $1 / 2-1 \mathrm{~g}$. This component logically reflects a combination of both displacement and rotational motion. Shifts in baseline, both abrupt and gradual, are discernible as changes in the order of $2 \mathrm{~g}$ (Component $\mathrm{B}$ ), lasting several seconds. This component, often of very low frequency, most probably represents $90-180^{\circ}$ rotations in the position of the transducers with respect to the vertical. A third component (C) consists of transient deflections, occasionally greater than $2 \mathrm{~g}$, which probably represent bumping of the transducer against the body; these deflections, in this damped system, contribute little to the overall power of the sampled signal.

\section{Frequency Characteristics of the Activity Signal}

Figure 3 depicts the averaged PSD functions from 105 68-sec data blocks. Very low frequency components (below about $0.25 \mathrm{~Hz}$ ) dominate the spectrum, and the contributions to power at frequencies above $1 \mathrm{~Hz}$ appear to be quite small. The first design problem is to assure that the transduction system is appropriately responsive to the frequency characteristics of the naturally occurring wrist activity signal.

\section{Desirable High-Pass Characteristics}

Because the wrist is restricted by the arm as to the distance it can travel, displacement accelerations which are very slow (low frequency) must also be very low in amplitude, and high-amplitude accelerations due to displacement must be of relatively high frequency. In contrast, rotational changes may be quite high (up to 2 g), irrespective of their frequency. If one assumes that displacement measures have more to do with the "energetics" of motility than mere rotation in the gravitational field, then it would seem desirable to separate displacement and rotational components. Although it is possible to do so using point-by-point analysis of triaxial data sets, such as in Figure 2, such analyses seem impractical for inexpensive portable devices, especially in the absence of empirical
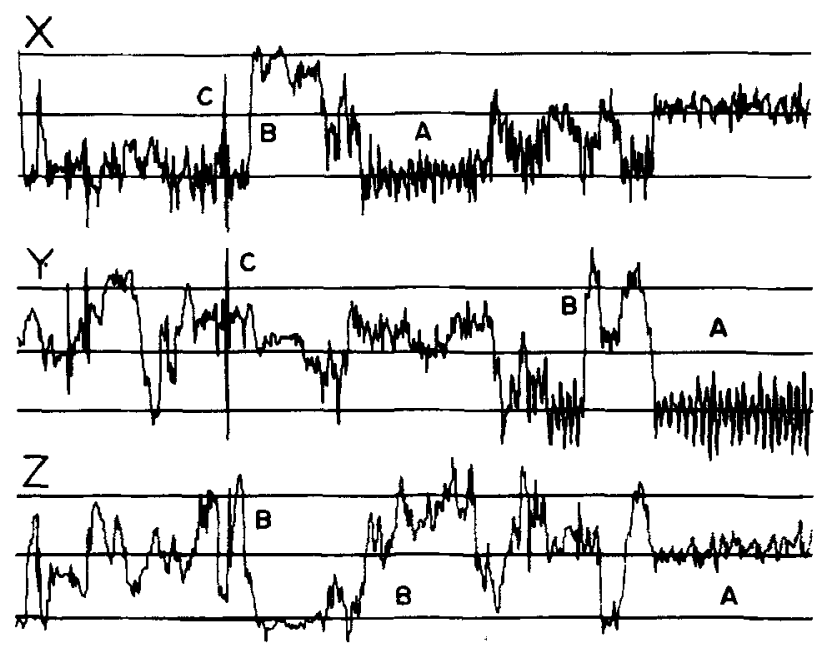

68 SEC

Figure 2. Raw wrist activity signal in three axes. Horizontal lines represent $\pm 1 \mathrm{~g}$ acceleration about zero. Passband $=$ dc $-8.3 \mathrm{~Hz}$, sampling rate $=15 \mathrm{~Hz}$. Components $\mathrm{A}, \mathrm{B}, \&$ C: see text. 


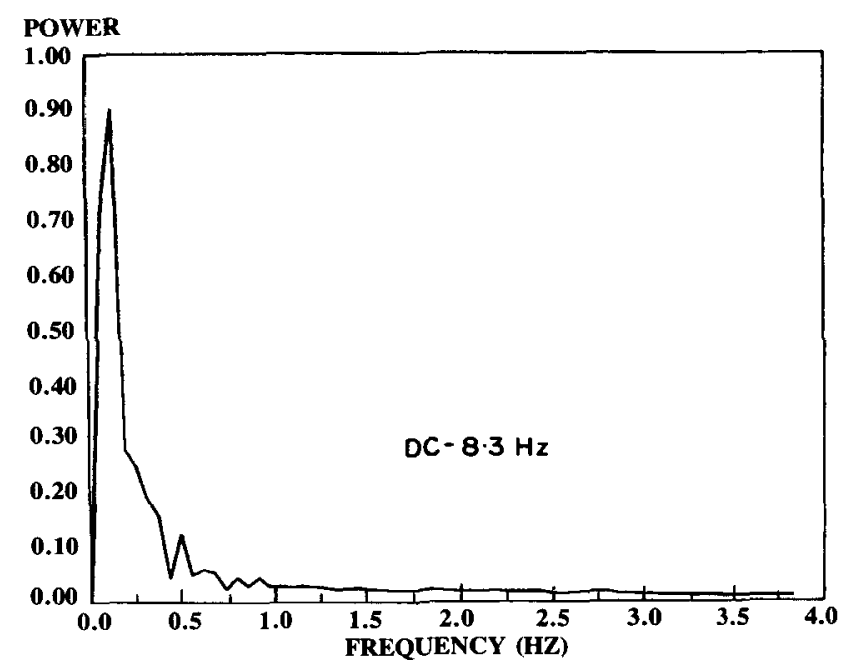

Figure 3. Averaged power spectral density (PSD) curve, from 105 68-sec blocks of raw signal. Passband $=\mathrm{dc}-8.3 \mathrm{~Hz}$, sampling rate $=15 \mathrm{~Hz}$.

evidence that it would be fruitful to do so (such evidence might be obtainable in a weightless state). Neither the arguments above, nor data in Figure 3, suggest that such a dissection is possible in the frequency domain, although the higher frequencies may favor the displacement component, with considerable overlap. Nevertheless, it still is desirable to reject the dc and very low frequencies, if only to prevent baseline shifts from "swamping" the detection of higher frequency movements. Wong et al. (1981), for instance, chose $0.125 \mathrm{~Hz}$ as a high-pass corner frequency, apparently with that rationale. Virtually all other devices described in the literature, in one way or another, are unresponsive to very slow movements; in most cases, the frequency response characteristics are not specified or even studied. For some devices (e.g., that of Colburn et al., 1976), the passband is estimated to be well above the area of interest (above $10 \mathrm{~Hz}$ ).

A pertinent basis for defining low-frequency rejection is a practical one. DC-responsive accelerometric strain gauges, of the type used in the studies reported here, are extremely expensive and difficult to fabricate in laboratory shops. With present technology, the appropriate choice for transducer elements has been to construct them from cantilevered piezoelectric ceramic plates or from bender elements (alternatives such as mercury switches are discussed further below). Such transducers are electrically capacitative (Wong et al., 1981): their lowfrequency response is restricted by trade-offs between physical size of circuit components, preamplifier input impedance and offset, and overall sensitivity. Thus, these practical considerations limit low-frequency response to approximately $0.1 \mathrm{~Hz}$.

There is a change in the curve's slope in the region of $0.25 \mathrm{~Hz}$ (see Figure 3); we chose to specify this design requirement to a range between $0.1-0.5 \mathrm{~Hz}$, with a target of $0.25 \mathrm{~Hz}$. The precise corner frequency realized, and its consistency across devices, is the subject of further study of the final product. Figure 4 represents the same 105-spectra average represented in Figure 3, after filtering the analog data through a high-pass filter set at $0.25 \mathrm{~Hz}$, with rolloff of $48 \mathrm{~dB} /$ octave.

\section{Desirable Low-Pass Characteristics}

The setting of the low-pass characteristic is selected to admit the majority of residual power (after high-pass filtration) of the activity signal for down-line processing or counting. According to Foster, McPartland, and Kupfer (1978), intentional human movements do not occur at a rate greater than about $200 / \mathrm{min}(3.3 \mathrm{~Hz})$, which might serve as a useful cutoff point. We assume that it is desirable to reject higher frequency movements, such as fine tremor and shivering, that occur in a range greater than 5 movements/sec.

Low-pass filtering provides rejection of high-frequency components of the signal, not caused by movement, that are important potential sources of noise. Sensitivity to audiofrequency $(>50 \mathrm{~Hz})$ and electromagnetic noise (usually 50 or $60 \mathrm{~Hz}$ ) should be eliminated, along with transients associated with large amplitude rotations and impacts (Components B and C in Figure 2, respectively). Furthermore, transducers built from cantilevered piezoelectric plates or phonograph pickups pose a special problem. These devices, used by several investigators including ourselves, are not fluid damped and possess a mechanical resonance of less than a few hundred hertz (Wong et al., 1981). On excitation by movement or impact, a significant part of the signal consists of this resonant component, potentially swamping any down-line counting process. Thus, it is necessary to attenuate such internal transducer vibration signals by analog filtering

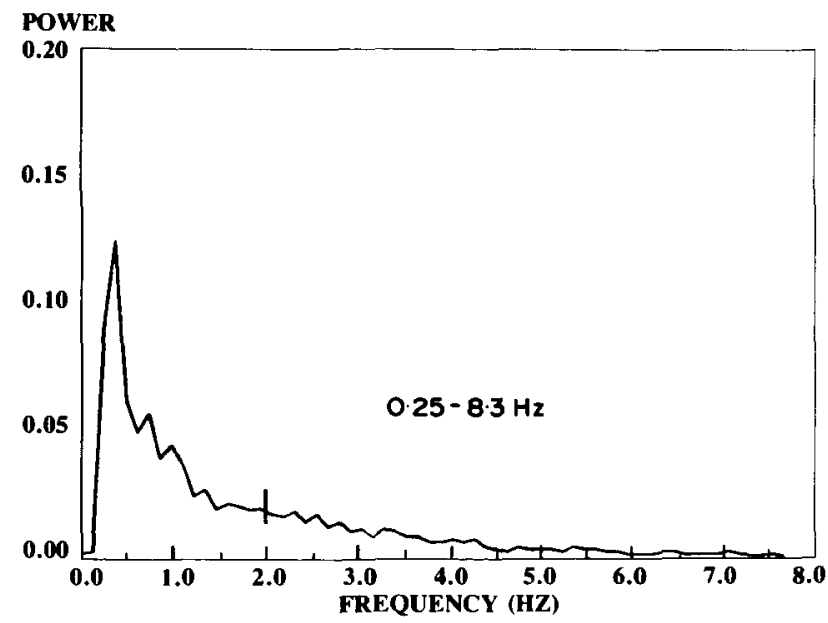

Figure 4. Power spectral density of highpass filtered data. Same as Figure 3. Passband $=0.25-8.3 \mathrm{~Hz}$. 
well below the resonant frequency of the transducer. Such filtering is absent from previous designs (Colburn et al., 1976; Kripke et al., 1978).

\section{External Vibration Artifact}

Contact of the transducer or the person wearing it with vibrating external objects is a source of noise. In our own applications, involving subjects riding in air and ground vehicles or operating machinery, vibration artifacts pose a major problem. With monitors of older design, we frequently could discern when the subject was driving a car, because the activity counters quickly reached maximum count. In the former designs, some of these artifacts were probably due to one transducer's resonant response discussed above, along with the absence of low-pass filtering, since the vibrations involved were well above $10 \mathrm{~Hz}$. However, when the frequency of vibration is near the range of natural human movements, the problem persists. Figure 5a exemplifies such artifacts: The subject first shook his wrist as fast as he could (about $4 \mathrm{~Hz}$ ), and then immediately grasped the handle of a power lawn mower (about $5.5 \mathrm{~Hz}$ ). Although the PSD curve (Figure 5c) clearly discriminates between the two components, lowpass filtering set to admit the movement component did not entirely eliminate the vibration artifact (Figure 5b).
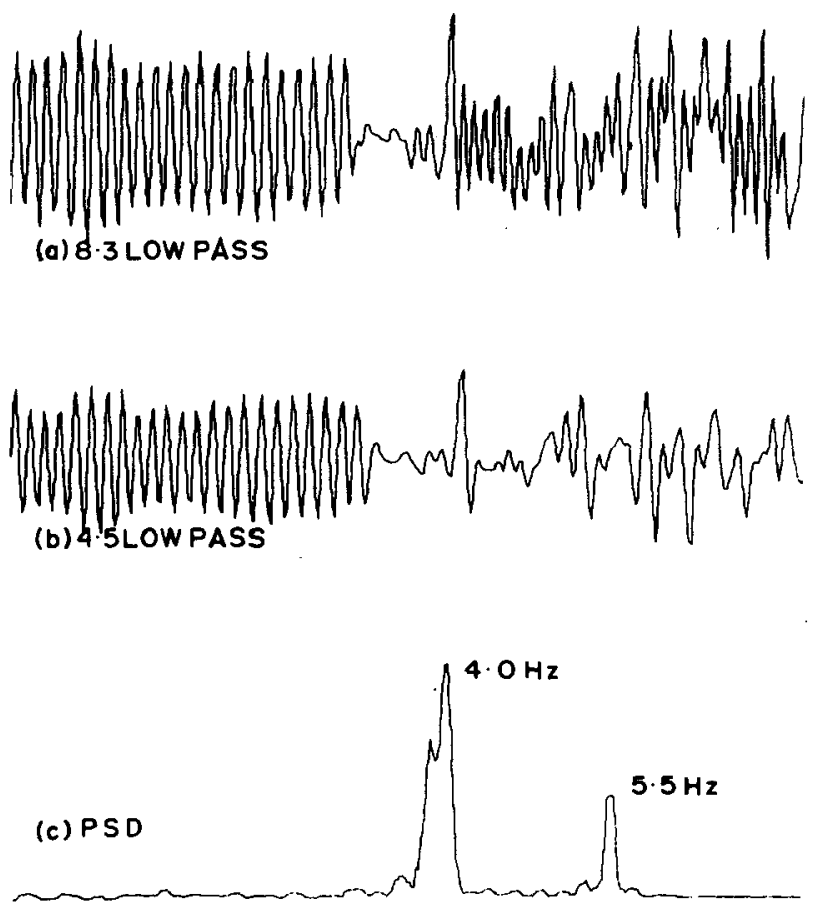

Figure 5. Low-pass filtering of vibration artifact. (a) The signal in the left portion is due to intentional shaking of the wrist, which is immediately followed by grasping a power lawn mower. (b) The same signal after low-pass filtering at $4.5 \mathrm{~Hz}$ (c) PSD function of data in (a).
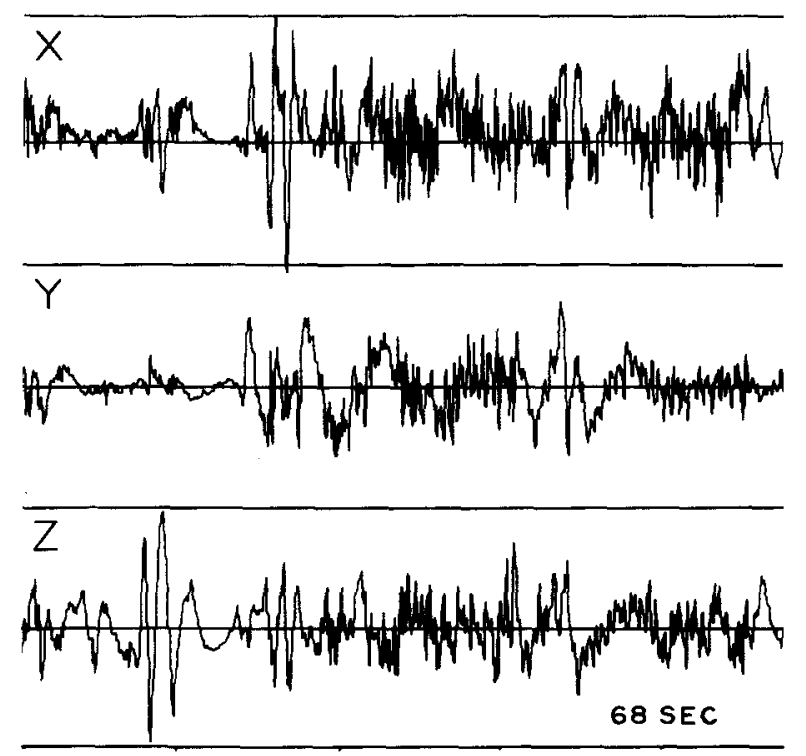

Figure 6. Band-passed filtrate of signal. Passband $=0.25-2.0 \mathrm{~Hz}$. Horizontal lines represent $\pm 1 \mathrm{~g}$ acceleration about zero.

\section{Selection of Low-Pass Cutoff Frequency}

Since the 4-Hz movements shown in Figure 5 represent a rare extreme, and because vibrational artifacts appear potentially more harmful than the loss of high-frequency data, a cutoff of $2 \mathrm{~Hz}$ was chosen for our specification, yielding a final passband of $0.25-2 \mathrm{~Hz}$, and accounting for more than $80 \%$ of the power displayed in Figure 4. Figure 6 displays a segment of the signal similar to that in Figure 2, but subjected to analog bandpass filtering in the specified range. The reader will note that rotational signal shifts and transients are attenuated.

In the final design, frequency response characteristics may be altered by simple component changes. Consequently, several devices have been produced with higher frequency responses, up to about $5 \mathrm{~Hz}$, in order to examine further the problem of artifact sensitivity and rejection.

\section{Sensitivity, Dynamic Range, and Threshold}

In the 2-h recording shown in Figure 1, the maximum deflection observed, after filtering, was the equivalent of 1.2-g acceleration, and maximum RMS acceleration for any 68 -sec interval in the recording was about $0.4 \mathrm{~g}$. In other observations, deflections above $1.5 \mathrm{~g}$ are exceedingly rare and transient. With the transducer resting on a table top (no motion), the RMS level for this system is about $0.01 \mathrm{~g}$, representing a background noise figure for the analog tape recorder. A minimum sensitivity specification, indicating the threshold above which a deflection should be counted as movement and not noise, was chosen to be in the range of 0.05-0.10 g, to provide rejection of noise in the face of possible temperature drift 
of both the threshold level and any signal offset from zero. This range of $0.05-1.5 \mathrm{~g}$ indicates a dynamic range in the order of $15 \mathrm{~dB}$. In terms of displacement acceleration, $0.05 \mathrm{~g}$ represents a rather low movement acceleration of $50 \mathrm{~cm} / \mathrm{sec}^{2}$. In terms of rotation, a change of $0.05-\mathrm{g}$ would occur from a change of $20^{\circ}$ or less, depending on initial orientation of the transducer. The actual threshold level realized in the design has a modest effect on the fidelity of the A/D transform used, as will be described below. Furthermore, since these estimates are based on a relatively noisy analog system, parameters of sensitivity and threshold are treated as adjustable variables in the digital design to permit further study.

\section{Directionality of Motion Transduction}

It would seem advantageous to use transducers sensitive in more than one direction, using three axes if possible. Figures 1 and 6 indicate that the instantaneous accelerations in axes $x, y$, and $z$ are quite different from one another. For the single piezoelectric bender element, the use of a mass weight offset from the central axis, as described by Wong et al. (1981), provides some sensitivity outside the bending axis, due to tortional movements of the plate. However, using a multiaxis sensing scheme, Webster et al. (1982) showed, at least grossly, that the signal from one axis was about as good as another. Our data confirm this. Movement detection does not occur in the instantaneous sense, but is counted or accumulated over a period of time; thus, data from each axis are summated over each sampling epoch, reducing the difference between axes. Figure 7 indicates that this reduction is a function of epoch length, so that by 1 sec or so, each axis provides a fair index of the signal in the others. This indicates that any given movement only rarely occurs purely in a single axis, and that within seconds a countermovement involving components in other axes occurs. Only sustained, repetitious movements in a single axis might escape detection in a monoaxial system. The ability to use one axis of motion sensing considerably reduces the expense, space, and complexity of electronic design of the specified system. Additional study, using the triaxial analog-recording system in direct comparison with the monoaxial digital system, is necessary to validate that important proposition in a wide variety of applications.

\section{Nonlinear Motion Sensors}

The use of a piezoelectric accelerometer has been emphasized largely because such a transducer provides a linear transformation of physical movement into an electronic signal and, thus, lends itself to analog processing, filtration, calibration, and adjustment using standard linear circuitry. The overall system relating movement to signal is very complex, involving the central and motor nervous systems, the elasticity of skin and tissue beneath the wrist-worn monitor, and the rigidity and mass of the case strapped to the wrist, and the mechanical and electrical characteristics of the transducer. Such a system is probably replete with sources of distortion and nonlinearity. Relationships between the raw signal and higher order measures of physiology and behavior, such as psychomotor activation or energy consumption, depend on empirical data, which is very meager at present.

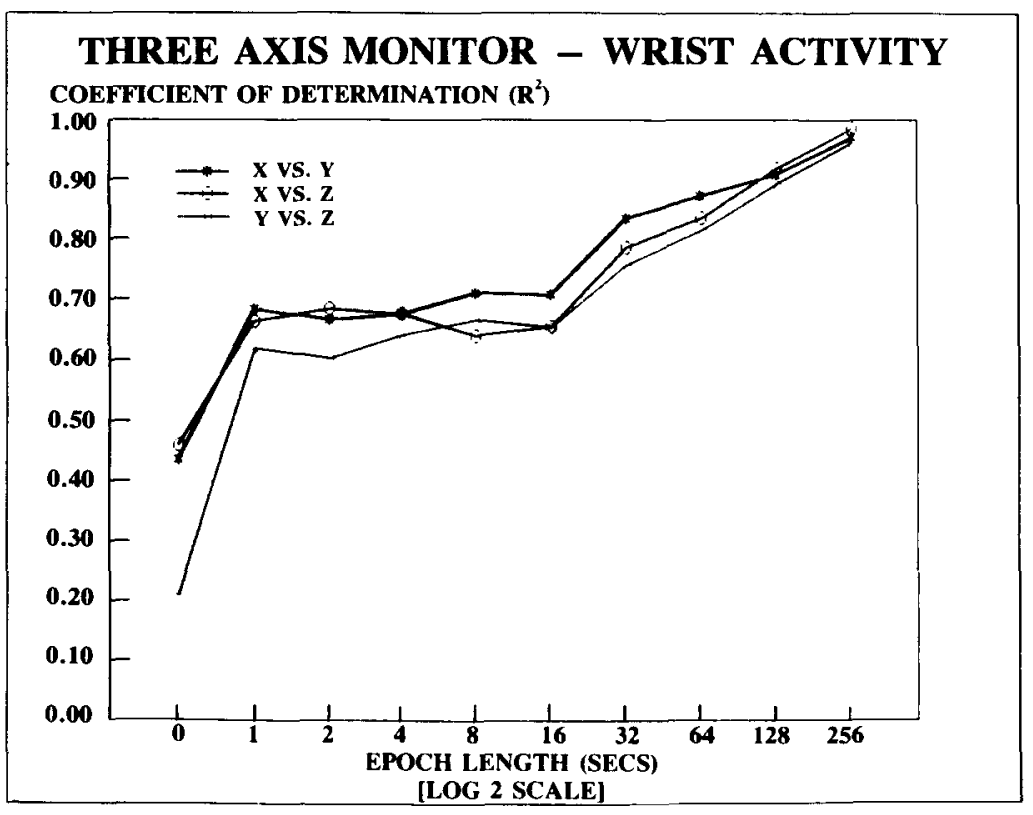

Figure 7. Interaction of triaxial signals as a function of epoch length. RMS values/epoch for each axis are linearly correlated with those of other axes at and above epoch $=1$ sec. Passband $=0.25-2.0 \mathrm{~Hz}$, sampling $=15 \mathrm{~Hz}$. 
Nevertheless, it seems parsimonious to maintain simplicity of the movement/signal transform to any extent possible. Other transducer systems have been proposed and implemented which are not simple, but instead introduce an additional response subsystem (nonlinear in concept) to an already complex system. These transducers have in common a mass which moves freely within a confined space or chamber: either a self-winding watch movement (Bell, 1968), a metal ball (Foster et al., 1972; Gueuning \& Eugene, 1985), or a globule of mercury (McPartland et al., 1976: Taylor et al., 1982). Movement of the arm produces a movement of the object, which, in turn, excites a transduction system or closes a switch. Thus, the movement-to-count transformation depends on the inertia of the mass; its size, shape, and elasticity; and, in the case of mercury, viscosity, surface tension, and the presence of impurities. Also involved are the dimensions and material of the containing chamber, the pathway of movement, and tendency of the mass to bounce, richochet, or vibrate. In any event, it seems unlikely that such systems are linearly responsive to a wide range of movements, in terms of amplitude and direction. The linear calibration curves of Foster et al. (1978) hold for repetitious, undirectional movements, so that counts are directly proportional to the number of movements; Foster et al.'s figures show a nonlinear relationship to the amplitude of movements.

Mercury-switch transducers (Taylor et al., 1982) have been disappointing, showing little or no response to lowamplitude movement (a variable threshold effect), high sensitivity to vibration artifact, and spontaneous oscillation. Comparison to linear accelerometers, by mounting both on the same wrist, reveals little relationship between the count of switch closures and the RMS level of activity. In 1060 -min repetitions, using 1-min epochs, the correlation coefficient between mercury-switch closures and RMS activity ranged from -0.10 to +0.40 ; Figure 8 displays one such 60 -min record, with null correlation be-
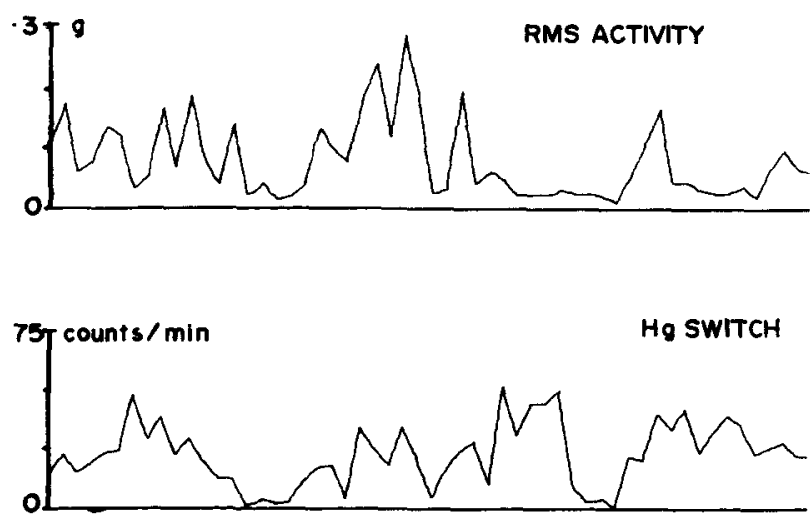

Figure 8. Comparison of mercury switch transducer and accelerometer. Mounted on the same wrist, 1-h data with 1 min epochs show variable sensitivity of the mercury switch, $r=0.00$.
RAW

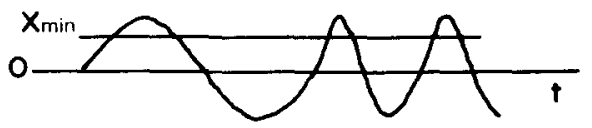

(a)

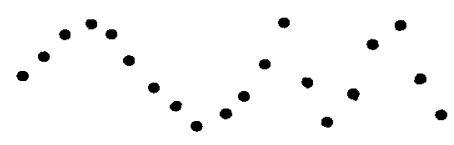

(b)

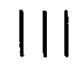

(c)

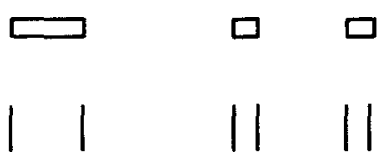

Figure 9. Analog to digital transforms of the signal. (a) A/D sampling at regular intervals. (b) Time-above-threshold (Xmin) based on regular sampling of comparator output. (c) Peak counting of each signal excursion above Xmin. (d) Threshold crossing detection, counting each excursion across $X \mathrm{~min}$.

tween the two transducers. These observations concur with those of Webster et al. (1982) and Wong et al. (1981).

\section{Analog-to-Digital Data Transformation}

Given an activity signal which has been bandpass filtered, there are four readily achieved methods for converting this continuous data into a form suitable for storage in digital memory format. The first of these involves ana$\log$ rectification and integration of the signal, with sampling and resetting of the integrator's output at the end of each epoch. Drift problems, component size, and power consumption tend to rule out this option, although it is utilized by Wong et al. (1981). The second method (Figure 9a) involves regular sampling (e.g., at $5 \mathrm{~Hz}$ ) within each epoch, with A/D conversion of the voltage waveform, followed by computation of some summary parameter (e.g., RMS level) at the end of each epoch. The versatility offered by the choice of computational algorithms and the linear relationship between the signal and the sampled time series make this a highly desirable choice. Unfortunately, micropower, miniature A/D converter circuits are not yet available, and the power drain of present circuits would sharply limit battery life.

The last two options involve the use of micropower comparator circuits, which test the signal against some reference voltage level and provide a binary output. The third choice consists of peak counting: counting the number of times per epoch that the signal level exceeds and then recedes below the reference threshold (Figure 9C). A variant of this method is threshold crossing detection, which provides a count twice that of peak counting 
(Figure 9d). The fourth option is similar, but by regular sampling (e.g., at $5 \mathrm{~Hz}$ ), it counts the time-abovethreshold (TAT) of the waveform (Figure 9b).

Because of the lower current consumption involved, implementation of the last two methods is favored in the present design. Given a comparator with the proper reference level, both methods are programmable; that is, the activity monitor's microprocessor can control the way the comparator output is managed (counted or sampled). The data transformation is not purely linear in relation to the signal, since a threshold level is imposed. Furthermore, peak counting clearly favors the higher frequency components of the signal, and time-above-threshold favors the lower frequencies. The pertinence of this distinction is not supported by empirical data as yet, although the timeabove-threshold method is possibly more desirable for use in environments with large amounts of vibrational noise.

Digitized data from the analog activity recorder were treated with algorithms which modeled these two digital transformations, and the results for a series of 68 -sec epochs are plotted in Figure 10. Linear regression of each series with the original signal-RMS series produced correlation coefficients plotted in Figure 11 as a function of the threshold level. Note that the time-above-threshold count provides the superior index of signal RMS level, which is expected since the greater power contribution is in the lower frequencies (see Figure 4). Both indices improve as the threshold rises above underlying noise levels.

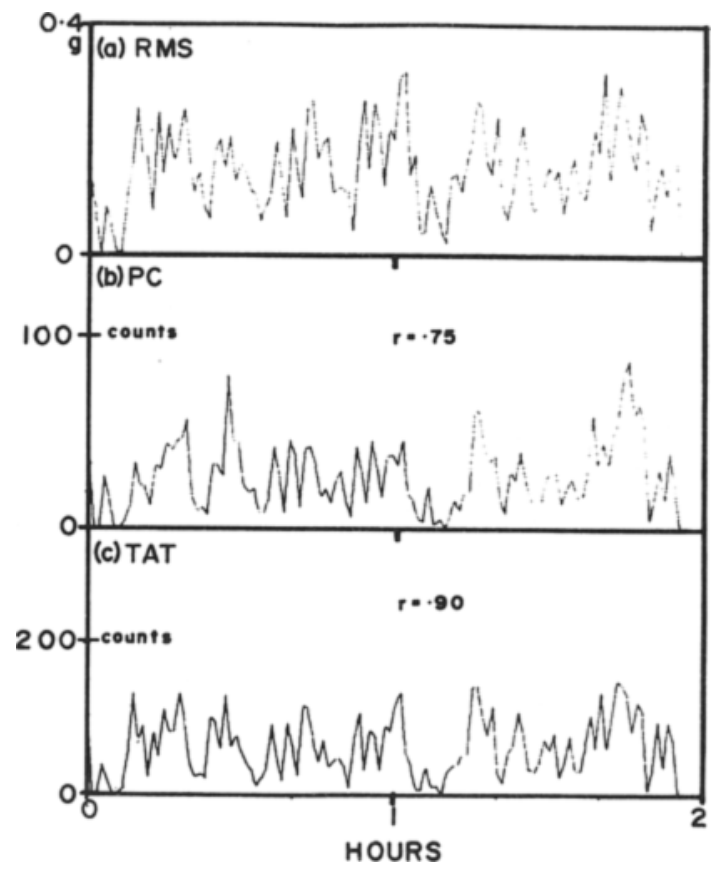

Figure 10. Comparison of digital counting transforms with RMS activity levels. Peak counting (b) and time-above-threshold (c) methods are plotted with RMS data (a) derived from same 2-h signal used in Figure 1.

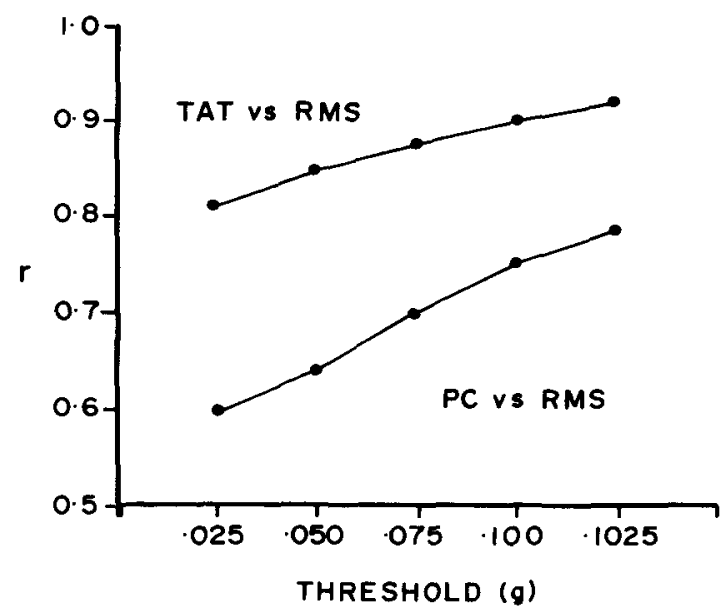

Figure 11. Linear correlation of digital counting transforms with RMS activity, plotted against $X$ min threshold level (see Figure 9 and text). Same signal data as in Figure 10. Correlation improves as Xmin increases above noise levels. TAT is the superior index of signal power.

These particular curves in Figures 10 and 11 are specific to the tape recording system used and its associated noise. Similar empirical determinations shall be made for the newly designed digital system. The activity-monitoring system has been specified to provide an adjustable threshold level, and a programmable choice between peak counting and time-above-threshold methods of data transformation, allowing further investigation of these transforms and their relationships to signal power and other physiological measures.

\section{Calibration}

Calibration of the activity index counter is achieved by applying a selected set of repetitious accelerations to the device, and serves to assure comparability from monitor to monitor. From several choices, we selected a pendulum with adjustable periods ranging from $2 \mathrm{sec}$ to $.5 \mathrm{sec}$ $(.5-2 \mathrm{~Hz})$, so that response within the bandwidth of interest may be recorded. The device is mounted with its sensitive axis perpendicular to the arc of the pendulum's swing. For each frequency tested, the analog waveform output is compared to calculated values of the radial component of acceleration along that arc. At manufacture, the analog circuitry is trimmed to provide consistent signals and a preset threshold. A recording is made of counter response to pendulum excursions. The point of pendulum decay at which counting ceases is a measure of the comparator threshold setting.

\section{Digital Dynamic Range and Memory Capacity}

In older activity monitors, the count-per-epoch was limited to 255 (an 8-bit binary word). Since counts were admitted at a rate of up to $10 \mathrm{sec}$, it often happened that for epochs longer than $25 / \mathrm{sec}$, counters became saturated. The resulting "topping off' effect produced rectangular waveforms in the activity time series, which in turn 
created difficulties for various time series' analytic methods.

In the design discussed here, the use of a microprocessor within the activity monitor permits storage of data on a bit-by-bit basis. Word length, in bits, is determined by the maximum count rate and the epoch length. Since the low-pass cutoff frequency is $2 \mathrm{~Hz}$, a $4-\mathrm{Hz}$ Nyquist sampling frequency is imposed; as it turns out, a 5-Hz counting frequency is more conveniently derived from the system clock, so this is the frequency used. Thus, the maximum number of counts is 300 (or 9 bits) in a 1-min epoch, 3,000 (or 12 bits) for a 10-min epoch, and so on up to 16 bits which permits a count of 65,535 (implying an epoch of 13,107 sec, or $3 \mathrm{~h}, 38 \mathrm{~min}$ ). Word length, in bits, and epoch length, in seconds, are specified to be separately programmable; minimum word length is set at 8 bits. If activity levels are expected to be relatively low, word length can probably be safely halved (reduced by one bit).

The total number of epochs which can be stored in the system is limited by RAM capacity, or battery life, whichever depletes first. Operating battery life is estimated at $1,000 \mathrm{~h}$ for the circuits used, and data collection periods of up to $720 \mathrm{~h}$ (or 30 days) are envisioned. The RAM capacity is $16 \mathrm{~K}$, or 131,072 bits. The various relationships are best summarized in the following equations, expressed in BASIC computer language:

(1) For word length in bits, and epoch length in seconds,

WORDLENGTH $=1+$ INT $($ LOG $(5 *$ EPOCH +1$)$

/ LOG( 2 ): IF WORDLENGTH $<8$ THEN WORDLENGTH $=8$.

(2) Given word length, then the length of the data Series in epochs is

$$
\text { SERIES = INT }(131072 / \text { WORDLENGTH }) \text {. }
$$

(3) The duration of data collection, in days, is given by

$$
\text { DAY }=\text { SERIES * EPOCH / ( 60/1440), }
$$

which should not exceed 30 days in practice.

\section{THE WALTER REED ACTIVITY MONITORING SYSTEM}

Figure 12 is a block diagram of the monitoring system developed according to the foregoing considerations and specifications. Specific design details, refinements, software development, and production were achieved by the

\section{MONITOR}

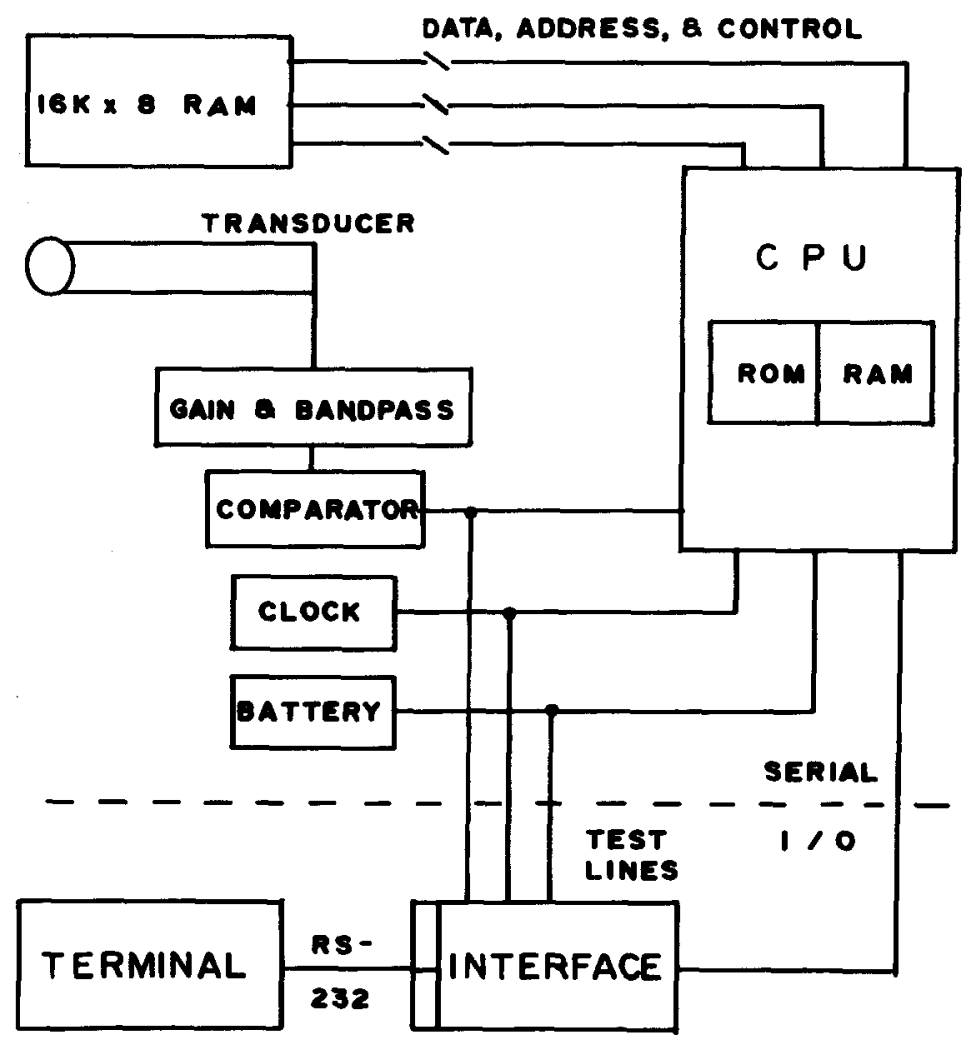

Figure 12. Simplified block diagram of the Walter Reed Activity Monitoring System. 

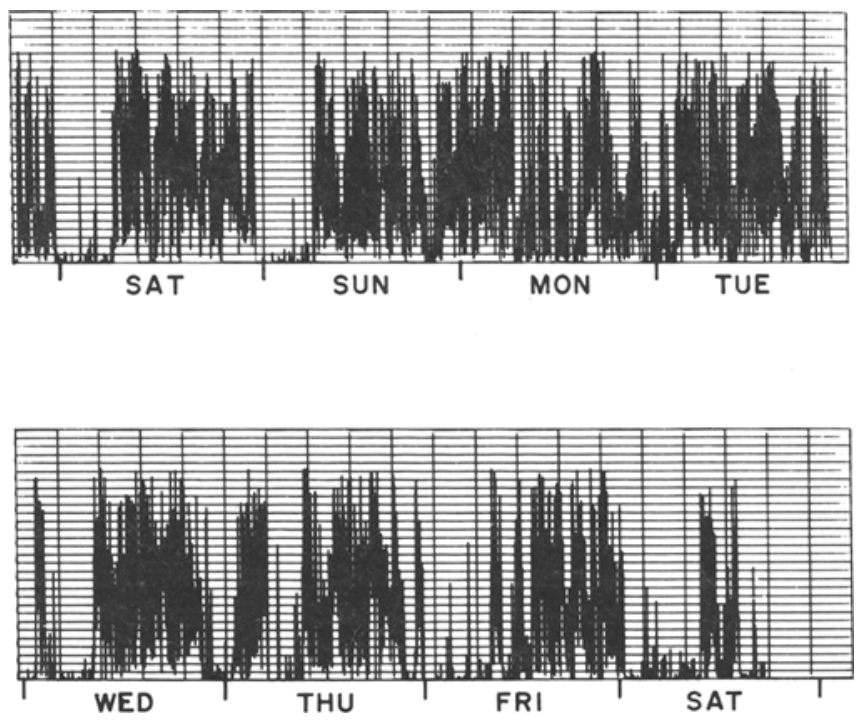

Figure 13. Activity of soldier in continuous field operation. Three-minute epochs, maximum count (vertical scale) $=255$.

contractors (Precision Control Design, Inc., Ft. Walton Beach, FL). The system uses a MC1468705G2L (Motorola) microprocessor with self-contained ROM for process and timing control, data sampling, and storage to external RAM. External communication is achieved by a serial line with RS232 8-bit format, using standard MODEM/XMODEM error checking protocol implemented by the on-board microprocessor, and brought to full RS232C voltage levels by the outboard serial data PC interface. Serial communication at 9600 baud is used to both initialize the monitor, and to "dump" the contents of RAM to the awaiting microcomputer, terminal, or printer. Initialization includes the insertion of clock time and date to the monitor's on-board clock, start/stop time and date, epoch length, data word length (in bits), counting method, and identification header. Data may be dumped either in 8-bit binary or hexadecimal formats (irrespective of data word length), or may be processed according to data word length, and dumped as a table of 5-digit decimal characters (a slower process but suitable for printer or screen listings).

Figure 13 presents an 8-day time series acquired with a prototype of the monitor. Epoch length was $180 \mathrm{sec}$, and data word length only 8 bits, so that occasional saturation (count of 255) of the epoch counter occurred. The subject was a soldier on a week's sustained combat training operation; fractionation of sleep is particularly notable in the early part of the week shown.

\section{DISCUSSION}

In their review of research on human activity, Cromwell, Baumeister, and Hawkins (1963) argued that there was a certain futility in viewing activity as a unitary, homogeneous phenomenon. Theory-based definitions of activity are as diverse as the means of measuring activity, and interrelationships even among reliable measures, or among useful theories, are weak, fragmentary, or unconfirmed. The authors state that "a precise definition of activity depends invariably on how it is measured." The statement in part conveys a warning about the limits on interpreting activity data acquired with pragmatic techniques, such as those discussed here, a caveat echoed by Webster et al. (1982, p. 744). The statement also urges that, if activity phenomena are to be carefully studied, definitions of the measures themselves should be precisely drawn. This approach is rarely taken in the design of instruments for monitoring movement and motility. For instance, should human activity be defined simply as displacement and rotational movement of a portion of the body, or as a complex disturbance of the environment involving movement, environmental reaction, and noise collectively? In the latter case, nonlinearities and broad-band sensitivity of detectors are acceptable, even desirable; however, this intent is rarely discussed (see Guening \& Eugene, 1985).

It is clear that activity monitors are useful in several pragmatic applications. By comparison with EEG data, Mullaney, Kripke, and Messin (1980) were able to estimate sleep time, sleep period, and wake after sleep onset with high reliability. During daytime activity, quantitative movement counts have been shown to correlate with residual drug effects (Crowley \& Hydinger-MacDonald, 1979), serum creatine phosphokinase (Goode, Meltzer, Moretti, Kupfer, \& McPartland, 1979), school achievement and self-image in children (Foster, McPartland, \& Kupfer, 1977), manic-depressive state (Wehr et al., 1983), occupational activity (LaPorte et al., 1982), and estimated energy expenditure (LaPorte et al., 1979).

Although Wong et al. (1981) did not explicitly calculate superior correlations between objective energy consumption measurement (oxygen consumption rate) and an 
activity measure, the correlations apparently are achieved when the latter takes into account the amplitude as well as the frequency (movement count) of activity. In order to record the intensity of movement, it is necessary to reduce the spurious contributions to signal power of nonlinearities and noise. This is the approach we have taken, emphasizing a narrow, linear band of sensitivity based on empirical measurements of wrist movements. The final specifications given our contractor involve some practical trade-offs and a certain amount of arbitrariness in defining the design parameters. On the other hand, the response characteristics and the $A / D$ transform of the monitor are well defined and treatable as variables: adjustments to the measurement system can be made readily on the basis of further study. This was our main objective. One problem remaining is that the choice of location, the nonpreferred wrist, is mainly one of convenience. Although other researchers have shown that this location provides a reliable index of gross activity in other areas of the body (Foster et al., 1978; Webster et al., 1982), our observations should be expanded.

Along with the feasibility of software and component adjustments, the new activity monitor adapts itself well to future modification in two key areas: (1) the use of truly linear signal processing through A/D conversion, and (2) the addition of other analog signal channels, such as electrocardiography and thermography, to monitor important correlates of activity and sleep. Finally, when reliability and relevance of design features are firmly established, present technology will permit drastic miniaturization and reduction in cost.

\section{REFERENCES}

BELL, R. Q. (1968). Adaptation of small wrist watches for mechanical recording of activity in infants and children. Journal of Experimental Child Psychology, 6, 302-305.

Borbeley, A. A., Loepfe, M., Mattman, P., Tobler, I. (1983). Midazolam and triazolam: Hypnotic action and residual effects after a single betime dose. Arzneimittel Forschung-Drug Research, 33, 1500-1502.

Colburn, T. R., Smith, B. M., Guarini, J. J., \& Simmons, N. N. (1976). An ambulatory activity monitor with solid state memory. ISA Transactions, 15, 149-154.

Cromwell, R. L., Baumeister, A., \&awkins, W. F. (1963). Research in activity level. In N. R. Ellis (Ed.), Handbook of mental deficiency (pp. 632-662). New York: McGraw-Hill.

Crowley, T. J., \& Hydinger-MacDonald, M. (1979). Bedtime flurazepam and the human circadian rhythm of spontaneous motility. Psychopharmacology, 62, 157-161.

Foster, F. G., KuPfer, D., Weiss, G, , LipPonen, V., McPartland, R., DELGADO, J. (1972). Mobility recording and cycle research in neuropsychiatry. Journal of Interdisciplinary Cycle Research, 3, $61-72$.

Foster, F. G., McPartland, R. J., Kupfer, D. J. (1977). Telemetric motor activity in children. Biotelemetry, 4, 1-8.

Foster, F. G., McPartland, R. J., \& KuPfer, D. J. (1978). Motion sensory in medicine, part I. A report on reliability and validity. Joumal of Inter-American Medicine, 3, 4-8.

Goode, D. J., Meltzer, H. Y., Moretti, R., Kupfer, D. J., \& MCPARTLAND, R. J. (1979). The relationship between wrist-mounted motor activity and serum CPK activity in psychiatric in-patients. British Journal of Psychiatry, 135, 62-66.

Guening, F., \& Eugene, C. (1985, March). A long-term microprocessor-based wrist-worn actimeter. Paper presented at the International Symposium on Ambulatory Monitoring, Padova, Italy.

Kripke, D. F., Mullaney, D. J., Messin, S., \& Wyborney, V. G. (1978). Wrist actigraphic measures of sleep and rhythms. Electroencephalography \& Clinical Neurophysiology, 44, 674-676.

Kupfer, D. J., Weiss, B. L., Foster, F. G., Detre, T. P., DelGado, J., MCPartland, R. (1974). Psychomotor activity in affective states. Archives of General Psychiatry, 30, 765-768.

LaPorte, R. E., Cauley, J. A., Kinsey, C. M., Corbett, W., Robertson, R., Black-Sandler, R., Kuller, L. H., \& Falkel, J. (I982). The epidemiology of physical activity in children, college students, middle-aged men, menopausal females, and monkeys. Journal of Chronic Diseases, 35, 787-795.

LaPorte, R. E., Kuller, L. H., Kupfer, D. J., McPartland, R. J., MAtThews, G., \& CASPERSEN, C. (1979). An objective measure of physical activity for epidemiologic research. American Journal of Epidemiology, 109, 158-168.

McPartland, R. J., Kupfer, D. J., \& Foster, F. G. (1976). The movement-activated recording monitor: A third-generation motoractivity monitoring system. Behavior Research Methods \& Instrumentation, 8, 357-360.

Mullaney, D. J., Kripke, D. F., \& Messin, S. (1980). Wristactigraphic estimation of sleep time. Sleep, 3, 83-92.

Porrino, L. J, , RAPoport, J. L., Behar, D., SCEery, W., ISMOND, D. R., \& BunNEY, W. E. (1983). A naturalistic assessment of the motor activity of hyperactive boys. Archives of General Psychiatry, 40, 681-687.

Taylor, C. B., Kraemer, H. C., Bragg, D. A., Miles, L. E., Rule, B., Savin, W. M., Debusk, R. F. (1982). A new system for long-term recording and processing of heart rate and physical activity in outpatients. Computers \& Biomedical Research, 15, 7-17.

Webster, J. B., Messin, S., Mullaney, D. J., \& Kripke, D. F. (1982). Transducer design and placement for activity recording. Medical \& Biological Engineering \& Computing, 20, 741-744.

Wehr, T. A., Sack, D., Rosenthal, N., Duncan, W., \& Gillin, J. C. (1983). Circadian thythm disturbances in manic-depressive illness. Federation Proceedings, 42, 2809-2814.

WEVER, R. A. (1981). On varying work-sleep schedules: The biological rhthym perspective. In L. C. Johnson, D. I. Tepas, W. P. Colquhoun, \& M. J. Colligan (Eds.), The twenty-four hour workday: Proceedings of a symposium on variations in work-sleep schedules (pp. 51-86). Cincinnati: National Institute of Occupational Safety and Health Publication No. 81-127.

Wong, T. C., Webster, J. G., Montoye, H. J., Washburn, R. (1981). Portable accelerometer device for measuring human energy expenditure. IEEE Transactions on Biomedical Engineering, BME$28,467-471$ 